\title{
Identification of Feed Resources for Cattle Fattening and Chemical Composition Determination in Dessie and Kombolcha Towns, Ethiopia
}

\author{
Kassahun Ahmed Seid (M.Sc) \\ College of Agriculture, Arba Minch University, P.O. Box 21 Arba Minch, \\ Ethiopia \\ Berhan Tamir (Professor, PhD) \\ Ashenafi Mengistu (Associate professor, PhD) \\ College of Veterinary Medicine and Agriculture, Addis Ababa University, \\ P.O. Box 34 Debre-Zeit, Ethiopia
}

\begin{abstract}
This study was designed to identify common conventional and nonconventional feed resources worth of feeding oxen for cattle fattening in and around Dessie and Kombolcha towns and evaluate the sources, availability, quantity, distribution, price and limitation of different feeds. Urban and peri urban kebeles who practices cattle fattening were totally considered in both study towns. Accordingly, 3 and 6 urban, 4 and 6 peri urban kebeles were selected from Dessie and Kombolcha towns, respectively. Complete enumeration techniques were applied to select urban and peri urban kebeles. Totally, 190 and 147 cattle fatteners were selected and interviewed in the identification process of feed resources used for cattle fattening practices in Dessie and Kombolcha towns, respectively. In both study towns 42 feed resources used for cattle fattening practices were identified. The identified feed resources were characterized in to different categories according to their nature and prioritized based on availability and abundance. Further feed resources categorized in to three groups based on their importance and level of significance for cattle fattening purpose, i.e., $1^{\text {st }}, 2^{\text {nd }}$ and $3^{\text {rd }}$ choice. From the totally identified feeds, nine major and highly significant feed resources, which were nominated by the respondents from both study towns, were selected and subjected for chemical analysis. The collected samples were examined to determine the DM, Ash, CP, NDF, ADF and ADL. In Dessie and Kombolcha towns, the identified cattle feed sources were poultry farms, flour factors, milling houses, micro and small food processors, local brewery houses, farmers, brewery factory, oil seed processers, pasture lands, feed traders, sugar processing factory, university and government aids. There was
\end{abstract}


high seasonal variability of feed availability and distribution in both study towns. September to December relatively there was abundant feed resources. On the other hand, the feed shortage and price increased severely towards January to June. As a result, storing and saving available cattle fattening feed resources during surpluses time is crucial for proper cattle fattening practices. In addition, the government intervention is recommendable to solve poor distribution, limited access of feed resources and to decreases transportation difficulties and costs.

Keywords: Conventional, cattle, feed, fattening, non-conventional

\section{Introduction}

The government of Ethiopia is trying to increase the export of ruminant livestock and meat that can contribute to market-led economic growth and poverty reduction in the country. One of the main constraints hampering this effort is the supply of cattle, sheep and goats which can meet export quality requirements (Adugna, 2007). Correspondingly, one of the major constraints to supply the indicated livestock is lack of feed, both in quality and quantity (Tilahun et al., 2005). Poor nutrition and feed shortages are root causes for the poor performance of the livestock sector in Ethiopia. Most livestock destined for export or slaughter are produced in the pastoral areas from rain fed pastures and are slaughtered with little or no access to better quality feeds required to increase weight, improve condition, dressing percentage and reduce age at slaughter. Poor nutrition results in slow growth rate in growing animals and low production and reproduction performance. Poorly fed fattening cattle take too long to reach optimum slaughter weight and the meat produced by such animals may not satisfy the desired quality attributes (such as tenderness) to fulfill the demand of the consumers. When the quality of the fodder is low, animals are not able to eat what is required to put on weight. Because of the slow growth rate, the animals become old before they reach the desired live weight for sale. Hence, the quality of the beef becomes far from satisfactory. In addition, feed utilization is very inefficient as most of the feeds (about 85\%) are used for body maintenance. In such a system there appears to be a tremendous potential for improvement.

Accordingly, current and up-to-date baseline information related to feed availability and quality is lacking in peri-urban areas under the prevailing situations. As a result, there is a need to investigate the feed demand and supply situation in the urban and peri-urban areas with the aim of identifying suitable strategies to provide adequate amounts and sufficient quality fodder to the animals (Zewdie, 2010). In addition, the availability of feed resources in the highlands of Ethiopia depends on the mode and 
intensity of crop production as well as population pressure (Seyoum et al., 2001). Generally, there is feed deficiency during dry seasons, and plenty of resource in other season (Firew and Getenet, 2010). On the other hand, there is a significant potential for feeding livestock from occasional surplus grains and agro industrial by-products such as oilseed cakes, milling by-products, molasses and feeds coming from poultry farms (poultry litter) and, brewery factories (spent grain). However, nutritive values and seasonal availability are not well documented (Adugna, 2007). Instead, in the current study towns, cattle fattening is one of the newly emerging activities under urban agriculture. The sector is generating employment opportunities and income generation for urban and peri urban dwellers. Particularly, cattle fattening is becoming an effective tool for poverty alleviation and an important business sector for those cattle fattening associations organized at small scale enterprise level and displaced farmers of urban and peri urban areas due to urbanization and industry zone establishment. Dependably, the sector is hindered by feed shortage, high prices and lack of available information regarding available feed resources. One of the most viable and simple management interventions to avert the gap (the severe feed shortage) is to identify the type, source, availability, quantity, distribution and price of locally available and accessible feed resources. Cognizant of this fact that, the current research was engrossed to alleviate information gap and to assess quality of selective feed resources used for fattening cattle in Dessie and Kombolcha towns. Accordingly, based on the above background, this study was designed to identify the availability, quantity, distribution and price of common conventional and non-conventional feed resources used for cattle fattening with their respective merits and demerits and to assess chemical composition of selective feed resources in Dessie and Kombolcha towns.

\section{Materials and Methods:}

\section{The Study Areas:}

The study was conducted in Dessie and Kombolcha towns. Dessie is located in northern part of Ethiopia in Amhara National Regional State, South Wollo Zone at a distance of $400 \mathrm{~km}$ from Addis Ababa, Ethiopia. Its astronomical location is at $11^{\circ} 8 \mathrm{~N}-11^{\circ} 46^{\prime}$ North latitude and $39^{\circ} 38^{\prime} \mathrm{E}$ 41013' East longitude. Relatively it is bounded by Kutaber Woreda in the north, Dessie Zuriya Woreda in the east, and by Kombolcha town in the south. The topography of Dessie is a highland type surrounded by 'Tossa' mountain (Dawit, 2013). Its elevation ranges between 2,470 and 2,550 meters above sea level (http://en.wikipedia.org/wiki/Dessie, retrieved in December 2014). Annual maximum and minimum temperatures of Dessie are $23.7{ }^{\circ} \mathrm{C}$ and $9{ }^{\circ} \mathrm{C}$, respectively, recorded in 2015 (Kombolcha meteorology station). Dessie is one of the reform towns in the region and has 
a city administration consisting of municipality, 10 urban and 6 peri urban kebeles. Kombolcha is an industrial town found in the north-central part of Ethiopia in South Wollo Zone of the Amhara Regional State of Ethiopia. It is situated at a distance of $377 \mathrm{~km}$ from north of Addis Ababa, $505 \mathrm{~km}$ from the Regional capital city, Bahirdar, $23 \mathrm{~km}$ from the zonal town Dessie and 533 $\mathrm{km}$ from port Djibouti. Astronomically, the town is located at about $11^{0} 6^{\prime} \mathrm{N}$ latitude and $39^{\circ} 45^{\prime} \mathrm{E}$ longitudes. The delimitation of the town is bounded by Dessie Zuria Woreda in the North East and North west, Kalu Woreda in the South and Albuko Woreda in the South West (Muluwork 2014). Mean annual rainfall is $1046 \mathrm{~mm}$ while annual maximum and minimum temperatures are $28.1{ }^{\circ} \mathrm{C}$ and $12.9{ }^{\circ} \mathrm{C}$, respectively, recorded in 2015 (Kombolcha meteorology station). The town is located in a range of altitudes between 1,500 and 1,840 $\mathrm{m}$ above sea level. Kombolcha is one of the reform towns in the region and has a town administration municipality, 5 urban and 6 peri urban kebeles. The city shares Kombolcha airport with its neighboring town, Dessie. Kombolcha is one of the few towns in Ethiopia with a relative greater number of large scale manufacturing plants including ELFORA-Meat Processing Factory, BGI-Brewery Factory and Flour Factories. On top of this, the town is selected to be an industrial zone by Amhara National Regional State and the Federal Government of Ethiopia (Eskinder et al., 2010).

\section{Sampling Procedure and Sample Size:}

Based on objectives of the research and the parameter required structured questionnaire was prepared. The questionnaire comprised data or information on types, quantity, seasonal availability, price, limitations, and users of different feed resources. Accordingly, those urban and peri urban kebeles where cattle fattening is practiced, were considered in both study towns for identification of feed resources used for cattle fattening. Accordingly, 3 and 6 urban, 4 and 6 peri urban kebeles were selected from Dessie and Kombolcha towns, respectively. Complete enumeration technique was applied to select urban and peri urban kebeles. Due to manageable number of cattle fatteners,' complete enumeration a technique was applied to select individuals from urban and peri urban kebeles, in the case of Kombolcha town. While, systematic random sampling technique for peri urban and complete enumeration technique for urban was applied to select individuals' in Dessie town. In the case of Dessie town, sampled households in peri urban kebeles were determined based on the principle of probability proportional to size'. Consequently, 190 and 147 cattle fatteners were selected totally and interviewed in the feed resources identification 
used for cattle fattening practices in Dessie and Kombolcha towns, respectively.

\section{Data Collection and Analysis:}

A single visit formal survey was employed to collect all the required data. To strengthen the survey data, group discussions were held with individuals who have knowledge and experience about common conventional and non-conventional feed resources used for cattle fattening. In addition, key informant interviews were made with towns and kebeles Agricultural Experts, Development Agents and different feed suppliers. Field observation was carried out to take different feed samples and pictures. Accordingly, focus group discussions and key informant interviews were conducted between February and April, 2016 whereas the household level surveys were carried out in May, June, July and August of the year 2016. As a result, common conventional and non-conventional feed resources (types), sources, availability, season of distribution, price/kg, current and overall production supply potential and different feed suppliers of governmental and private organizations, limitations and worthiness of feed resources used for cattle fattening in Dessie and Kombolcha towns were studied through structured questionnaires, key informant interviews, group discussions and field observation.

Representative feed samples from each study town were collected from different cattle fatteners and feed suppliers. In addition, during field observation and sample collection pictures were captured to strengthen the information obtained during survey, group discussion and key informants interviews. The feeds were categorized into conventional and nonconventional based on their nature. Again, conventional feed sources were further classified in to dry roughage, green roughage and concentrate categories. In addition, cattle fatteners prioritized feed resources based on availability and abundance. Furthermore, as per group discussion, cattle fatteners grouped the overall identified feed resources in to three categories, explicitly, $1^{\text {st }}$ choice, $2^{\text {nd }}$ choice, and $3^{\text {rd }}$ choice feed resources based on importance and significance for cattle fattening. Index was calculated to provide ranking of conventional and non-conventional feed resources according to the indicated formula. Index of conventional and nonconventional feed resources was calculated. First a weighted value was given for each feed based on their rank ( 3 for the $1^{\text {st }}$ feed, 2 for the $2^{\text {nd }}$ feed, and 1 for the $3^{\text {rd }}$ feed). The number of households' response for a particular feed was multiplied by a given weighted value. Then the rank was given by dividing the value of each feed to the total sum of all feeds value. 
Finally, based on the analysis result via considering the respondent response, availability of the feeds, abundance, distribution, accessible potential and significance of feed resources for cattle fattening from the totally identified feeds, nine major and highly significant feed resources which were score $1^{\text {st }}$ rank by the respondents from both study towns were selected and subjected for chemical analysis. Chemical analysis of the selected feeds was done at Holleta Agricultural Research Centre Nutrition Laboratory in Ethiopia. The collected samples were thoroughly mixed and enough sub-samples were taken to determine the DM, Ash, CP, Neutral detergent fiber (NDF), acid detergent fiber (ADF) and acid detergent lignin (ADL). Hemicellulose (HC) and cellulose (C), OM contents were calculated as NDF minus ADF, ADF minus ADL and DM minus Ash, respectively.

\section{Results:}

\section{Identified Feed Resources used for Fattening Cattle in Dessie and Kombolcha Towns}

In Dessie and Kombolcha towns totally 42 feed resources used for cattle fattening practices were identified (39 feeds in common from both study towns and 3 feeds uniquely from Kombolcha Those identified feed resources were used as major and supplementary feeds by the interviewed cattle fatteners which grouped further into three categories: viz; (i) green roughages, (ii) dry roughages and (iii) concentrates. Moreover, cattle fatteners prioritized the identified feed resources based on availability and abundance as presented in Table 1 and 2 
Table1: Identified roughage and non-conventional feed resources used for cattle fattening purpose in Dessie and Kombolcha towns with availability and

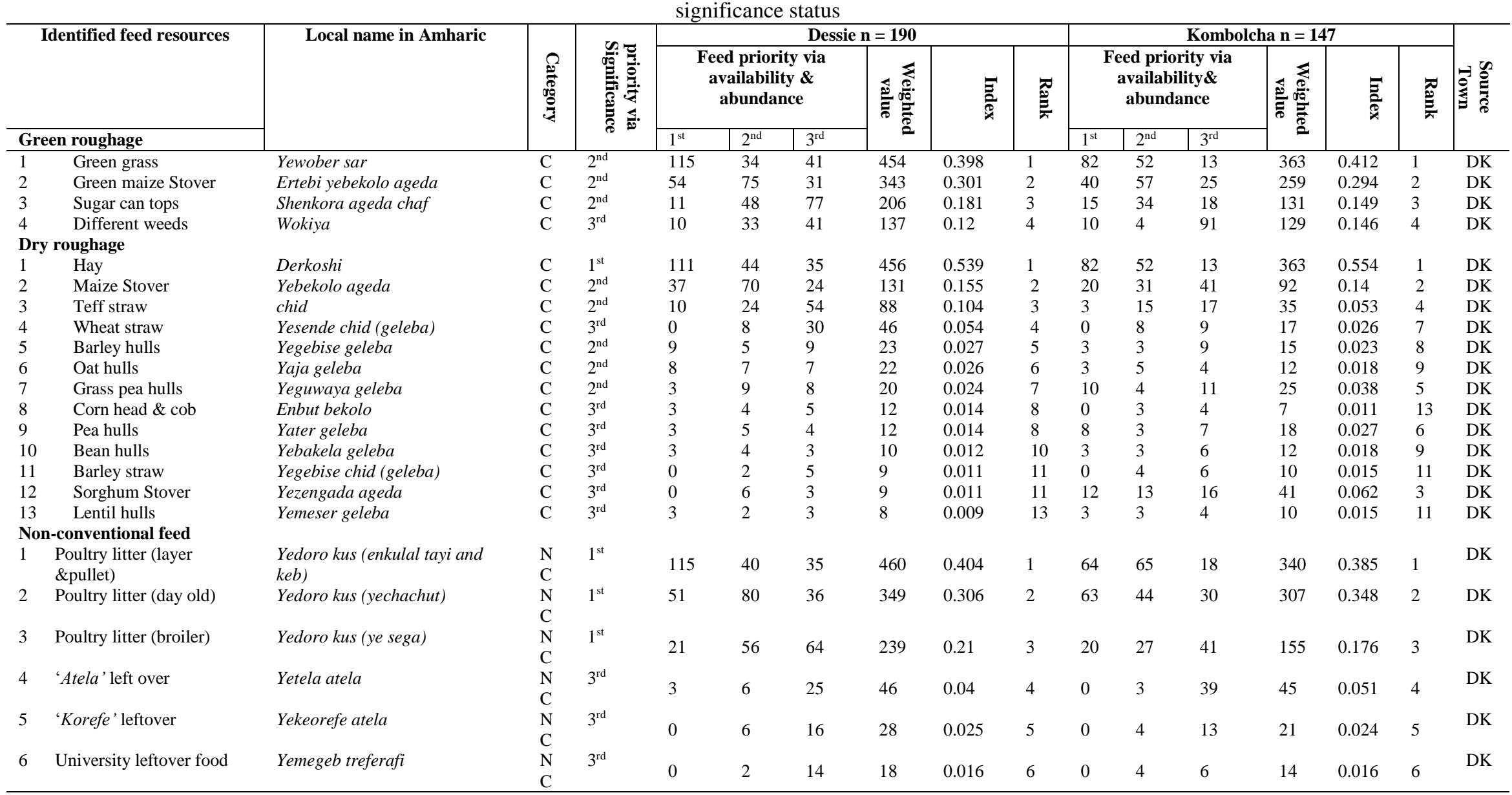

$\mathrm{Duka}^{\wedge}=$ leftover and occasional surplus or damaged grain during processing $\mathrm{C}=$ conventional NC non-conventional DK= Dessie and Kombolcha towns 
Table 2: Identified concentrate feed resources used for cattle fattening purpose in Dessie and Kombolcha towns with availability and significance status

\begin{tabular}{|c|c|c|c|c|c|c|c|c|c|c|c|c|c|c|c|c|c|}
\hline \multirow{3}{*}{\multicolumn{2}{|c|}{$\begin{array}{l}\text { Identified feed resources } \\
\text { (Concentrate) }\end{array}$}} & \multirow[t]{3}{*}{ Local name (Amharic) } & \multirow{3}{*}{ 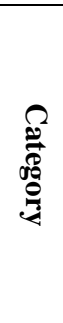 } & \multirow{3}{*}{ 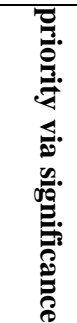 } & \multicolumn{6}{|c|}{ Dessie $n=190$} & \multicolumn{6}{|c|}{ Kombolcha $n=147$} & \multirow[t]{3}{*}{$=0$ w } \\
\hline & & & & & \multicolumn{3}{|c|}{$\begin{array}{c}\text { Feed priority via } \\
\text { availability \& } \\
\text { abundance }\end{array}$} & \multirow{2}{*}{ 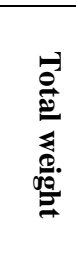 } & \multirow{2}{*}{$\overline{\bar{z}}$} & \multirow{2}{*}{ 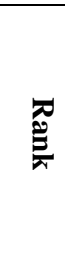 } & \multicolumn{3}{|c|}{$\begin{array}{c}\text { Feed priority via } \\
\text { availability \& } \\
\text { abundance }\end{array}$} & \multirow{2}{*}{ 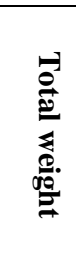 } & \multirow{2}{*}{$\overline{\bar{z}}$} & \multirow{2}{*}{ 률 } & \\
\hline & & & & & $1^{\text {st }}$ & $2^{\text {nd }}$ & $3^{\text {rd }}$ & & & & $1^{\text {st }}$ & $2^{\text {nd }}$ & $3^{\text {rd }}$ & & & & \\
\hline 2 & Corn grain & Bekole & $\mathrm{C}$ & $1^{\mathrm{st}}$ & 31 & 22 & 13 & 150 & 0.132 & 2 & 38 & 32 & 13 & 213 & 0.236 & 1 & DK \\
\hline 3 & Dried brewers grain & Derek yebira chimaki & $\mathrm{NC}$ & $1^{\text {st }}$ & 28 & 23 & 7 & 137 & 0.12 & 3 & 24 & 9 & 8 & 98 & 0.109 & 3 & $\mathrm{~K}$ \\
\hline 4 & Wet brewers grain & Ertib yebira chimaki & $\mathrm{C}$ & $1^{\text {st }}$ & 20 & 22 & 5 & 109 & 0.096 & 4 & 24 & 26 & 12 & 136 & 0.151 & 2 & $\mathrm{~K}$ \\
\hline 5 & Wheat middling & furshikelo & $\mathrm{NC}$ & $2^{\text {nd }}$ & 14 & 16 & 9 & 83 & 0.073 & 5 & 13 & 9 & 4 & 61 & 0.068 & 6 & DK \\
\hline 6 & Grass pea leftover & Yeguwaya duka $a^{\wedge}$ & $\mathrm{C}$ & $1^{\mathrm{st}}$ & 13 & 9 & 11 & 68 & 0.06 & 6 & 13 & 9 & 6 & 63 & 0.07 & 5 & DK \\
\hline 7 & Oat grain & Aja & $\mathrm{C}$ & $2^{\text {nd }}$ & 10 & 13 & 9 & 65 & 0.057 & 7 & 3 & 12 & 17 & 50 & 0.055 & 7 & DK \\
\hline 8 & Pea leftover & Yater duka^ & $\mathrm{C}$ & $1^{\text {st }}$ & 5 & 9 & 24 & 57 & 0.05 & 8 & 7 & 0 & 2 & 16 & 0.018 & 14 & $\mathrm{DK}$ \\
\hline 9 & Wheat leftover & Yesende duka $a^{\wedge}$ & $\mathrm{C}$ & $1^{\text {st }}$ & 0 & 8 & 30 & 46 & 0.04 & 9 & 0 & 8 & 9 & 25 & 0.028 & 9 & DK \\
\hline 11 & Linseed cake & fagulo ye telba & $\mathrm{C}$ & $2^{\text {nd }}$ & 5 & 7 & 14 & 43 & 0.038 & 11 & 0 & 2 & 0 & 4 & 0.004 & 18 & $\mathrm{DK}$ \\
\hline 12 & Different feed left over & Entiritaryi & $\mathrm{C}$ & $2^{\text {nd }}$ & 3 & 9 & 8 & 35 & 0.031 & 12 & 0 & 4 & 11 & 19 & 0.021 & 12 & DK \\
\hline 13 & Noug seed cake & fagulo ye nuge & $\mathrm{C}$ & $3^{\text {rd }}$ & 4 & 5 & 8 & 30 & 0.026 & 13 & 0 & 1 & 0 & 2 & 0.002 & 19 & DK \\
\hline 14 & Milling leftover & Yewoficho bet tiragi & $\mathrm{C}$ & $3^{\text {rd }}$ & 0 & 7 & 9 & 23 & 0.02 & 14 & 0 & 2 & 9 & 13 & 0.014 & 15 & $\mathrm{DK}$ \\
\hline 15 & Barley leftover & Yegebise duka^ & $\mathrm{C}$ & $2^{\text {nd }}$ & 3 & 5 & 4 & 23 & 0.02 & 14 & 4 & 3 & 7 & 25 & 0.028 & 9 & DK \\
\hline 16 & Bean leftover & Yebakela duka^ & $\mathrm{C}$ & $1^{\mathrm{st}}$ & 3 & 4 & 5 & 22 & 0.019 & 16 & 0 & 3 & 4 & 10 & 0.011 & 17 & DK \\
\hline 17 & Lentils leftover & Yemeser duka & $\mathrm{C}$ & $3^{\text {rd }}$ & 3 & 4 & 3 & 20 & 0.018 & 17 & 0 & 3 & 6 & 12 & 0.013 & 16 & $\mathrm{DK}$ \\
\hline 18 & Formulated ration & Yetemetatene meno & $\mathrm{C}$ & $1^{\text {st }}$ & 3 & 2 & 3 & 16 & 0.014 & 18 & 3 & 3 & 4 & 19 & 0.021 & 12 & $\mathrm{DK}$ \\
\hline 19 & Molasses & - & $\mathrm{C}$ & $2^{\text {nd }}$ & 0 & 6 & 3 & 15 & 0.013 & 19 & 3 & 5 & 16 & 40 & 0.044 & 8 & $\mathrm{~K}$ \\
\hline
\end{tabular}

$\mathrm{Duka}^{\wedge}=$ leftover and occasional surplus or damaged grain during processing $\mathrm{C}=$ conventional NC non-conventional $\mathrm{D}=\mathrm{Dessie}$ and Kombolcha towns $\mathrm{K}=\mathrm{Kombolcha}$ 


\section{Classifications of the Identified Feed Resources Based on Significance for Cattle Fattening in Dessie and Kombolcha Towns}

As per the group discussion, in both study towns cattle fatteners were categorized the overall identified 42 feed resources in to three categories depend on cattle fatteners day to day practical experience, explicitly, $1^{\text {st }}, 2^{\text {nd }}$, and $3^{\text {rd }}$ choice feed resources according to their importance and significance level for cattle fattening purpose as indicated in Table 1 and 2 .

\section{Potential Feed Suppliers:}

Major feed suppliers for cattle fattening practices were identified exhaustively in Dessie and Kombolcha towns. In addition, the type of feed or feeds each supplier produced, current as well as overall production capacity and marketing price were also evaluated. By and large, the information about the identified feed sources and current and overall production capacities of each feed sources were summarized and tabulated in Table 3 and 4.

Table 3: Major feed suppliers (flour factories) and production potentials of different feeds for fattening cattle in Dessie and Kombolcha towns

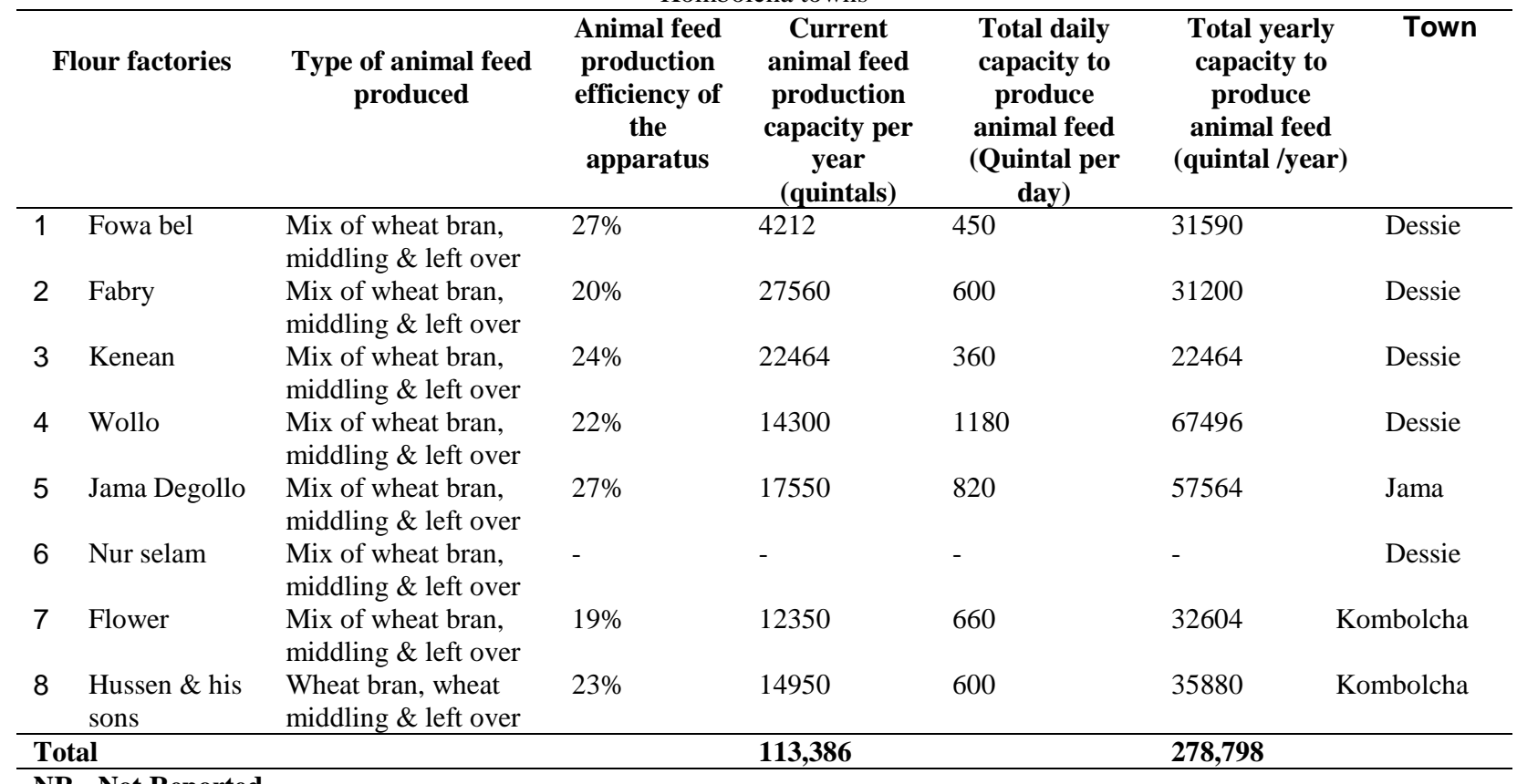

NR= Not Reported 


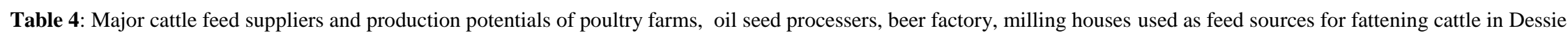
and Kombolcha towns

\begin{tabular}{|c|c|c|c|c|c|}
\hline \multicolumn{2}{|c|}{ Supplier organization } & Feed type & $\begin{array}{l}\text { Production } \\
\text { capacity/ month }\end{array}$ & Production capacity/year & Town \\
\hline \multicolumn{6}{|c|}{ Poultry farms } \\
\hline 1 & Kombolcha & Poultry litter & 126.3 quintals & 1515.31 quintals & Kombolcha \\
\hline 2 & Gerado & Poultry litter & 150 quintals & 1800 quintals & Dessie \\
\hline 3 & Bishoftu & Poultry litter & Access via traders & Access via traders & Bishoftu \\
\hline 4 & Small scale private farms & Poultry litter & - & - & Dessie \& Kombolcha \\
\hline \multicolumn{6}{|c|}{ Small scale oil seed processers (SSOSP) } \\
\hline 1 & Seid Ali SSOSP & Noug seed \& Linseed cake & - & Locked due to oil seed shortage & Dessie \\
\hline 2 & Huda SSOSP & Noug seed \& Linseed cake & 10 quintals & 120 quintals/year & Dessie \\
\hline 3 & Lemma SSOSP & Noug seed \& Linseed cake & - & Locked due to oil seed shortage & Kombolcha \\
\hline \multicolumn{6}{|c|}{ Beer factory } \\
\hline 1 & Kombolcha BGI ETHIOPIA & $\begin{array}{l}\text { Wet Brewers Grain and } \\
\text { Dried Brewers Grain }\end{array}$ & $130-150$ car & $1560-1800 \mathrm{car}$ & Kombolcha \\
\hline \multicolumn{6}{|c|}{ Milling house } \\
\hline 1 & $\begin{array}{l}34 \text { milling house in Dessie town ( } 15 \text { quintal per } \\
\text { each per month) }\end{array}$ & $\begin{array}{l}\text { Different feed Left over and } \\
\text { hulls }\end{array}$ & 510 quintals & 5100 quintals & Dessie \\
\hline 2 & $\begin{array}{l}39 \text { milling house in Kombolcha town (15 quintal } \\
\text { per each per month) }\end{array}$ & $\begin{array}{l}\text { Different feed Left over and } \\
\text { hulls }\end{array}$ & 585 quintals & 5850 quintals & Kombolcha \\
\hline \multicolumn{6}{|c|}{ Food processers at micro level } \\
\hline 1 & $\begin{array}{l}18 \text { small scale food processer in Dessie town ( } 12 \\
\text { quintal per each per month) }\end{array}$ & $\begin{array}{l}\text { Different feed Left over and } \\
\text { hulls }\end{array}$ & 216 quintals & 2160 quintals & Dessie \\
\hline 2 & $\begin{array}{l}36 \text { small scale food processor in Kombolcha (12 } \\
\text { quintal per each per month) }\end{array}$ & $\begin{array}{l}\text { Different feed Left over and } \\
\text { hulls }\end{array}$ & 432 quintals & 432 quintals & Kombolcha \\
\hline
\end{tabular}




\section{Marketing price of the identified feed resources:}

The marketing price of different feed resources is presented in Table 5, 6, and 7

Table 5: Identified non-conventional and green roughage feed resources used for cattle fattening practices, price, sources and users in Dessie and Kombolcha towns

\begin{tabular}{|c|c|c|c|c|c|c|}
\hline & Feed resources & Unit & $\begin{array}{c}\text { Current } \\
\text { price } \\
\text { (Birr) } \\
\end{array}$ & $\begin{array}{l}\text { Price } \\
\text { range } \\
\text { (Birr) }\end{array}$ & Sources & Users \\
\hline \multicolumn{7}{|c|}{ Non-conventional feed } \\
\hline 1 & Poultry litter from day old & $\mathrm{kg}$ & 2 & $2-4.5$ & $\begin{array}{l}\text { Dominantly from Kombolcha poultry farm, } \\
\text { sometimes form Dessie /Gerado/ and Bishoftu } \\
\text { poultry farm }\end{array}$ & Urban cattle fatteners \\
\hline 2 & Poultry litter from layers & $\mathrm{kg}$ & 2 & $2-4.5$ & $\begin{array}{l}\text { Dominantly from Kombolcha poultry farm, } \\
\text { sometimes from Dessie Gerado and Bishoftu } \\
\text { poultry farm }\end{array}$ & Urban cattle fatteners \\
\hline 3 & Poultry litter from broiler & $\mathrm{kg}$ & 2 & $2-4.5$ & $\begin{array}{l}\text { Dominantly from Kombolcha poultry farm, } \\
\text { sometimes from Dessie Gerado and Bishoftu } \\
\text { poultry farm }\end{array}$ & Urban cattle fatteners \\
\hline \multirow[t]{2}{*}{4} & 'Atela' left over & Per 25 litter & 15 & $10-20$ & Small scale home brewing in DK & Urban cattle fatteners \\
\hline & & Monthly rent & 250 & $200-300$ & Small scale home brewing in DK & Urban cattle fatteners \\
\hline \multirow[t]{2}{*}{5} & 'Korefe' leftover & Per 25 litter & 12.50 & $12-20$ & Small scale home brewing in DK & Urban cattle fatteners \\
\hline & & Monthly rent & 200 & $150-250$ & Small scale home brewing in DK & Urban cattle fatteners \\
\hline 6 & University leftover food & - & - & - & $\begin{array}{l}\text { Wollo University (Dessie and Kombolcha } \\
\text { campus) }\end{array}$ & $\begin{array}{l}\text { Nearest peri urban cattle } \\
\text { fatteners }\end{array}$ \\
\hline \multicolumn{7}{|c|}{ Green roughage } \\
\hline \multirow{2}{*}{$\begin{array}{l}1 \\
2\end{array}$} & Green grass & 'Besew shekim' & 65 & $50-80$ & Farmers farm & Peri urban cattle fatteners \\
\hline & Green maize Stover & 'Besew shekim' & - & - & - & $\begin{array}{c}\text { Peri urban cattle fatteners } \\
\text { mostly }\end{array}$ \\
\hline 3 & Sugar can tops & Per single can top & 0.25 & $0.25-1.00$ & DK sugar cane market & Urban cattle fatteners \\
\hline 4 & Different weeds & 'Besew shekim' & - & - & Farmers farm in DK & $\begin{array}{l}\text { Peri urban cattle fatteners } \\
\text { only }\end{array}$ \\
\hline
\end{tabular}


Table 6: Identified dry roughage feed resources used for cattle fattening practices, price, sources and users in Dessie and Kombolcha towns

\begin{tabular}{|c|c|c|c|c|c|c|}
\hline \multicolumn{2}{|c|}{$\begin{array}{l}\text { Dry roughage feed } \\
\text { resources }\end{array}$} & Unit & \multirow{2}{*}{$\begin{array}{c}\begin{array}{c}\text { Current } \\
\text { price (Birr) }\end{array} \\
100\end{array}$} & \multirow{2}{*}{$\begin{array}{c}\begin{array}{c}\text { Price } \\
\text { range } \\
\text { (Birr) }\end{array} \\
90-120\end{array}$} & Sources & User \\
\hline 1 & Hay & Donkey capacity & & & Farmers grazing land in DK & Peri urban and urban fatteners \\
\hline & & Car capacity (Isuzu) & 3000 & $2500-3000$ & From Chefa and Kemisse area & Urban cattle fatteners \\
\hline & & Per piled & 55 & $50-60$ & From Chefa and Kemisse area & Urban cattle fatteners \\
\hline 2 & Maize stover & Per 'Kemer' & 300 & $300-500$ & Farmers farm in DK & $\begin{array}{l}\text { Peri urban cattle fatteners } \\
\text { dominantly }\end{array}$ \\
\hline 3 & Teff straw & Donkey capacity & 170 & $150-200$ & Farmers farm + market in DK & $\begin{array}{l}\text { Peri urban cattle fatteners } \\
\text { dominantly }\end{array}$ \\
\hline & & Per piled & 85 & $47-85$ & Market in DK & urban fatteners \\
\hline 4 & Wheat straw & Donkey capacity & 160 & $140-200$ & Farmers farm in DK & $\begin{array}{l}\text { Peri urban cattle fatteners } \\
\text { dominantly }\end{array}$ \\
\hline 5 & Barley hulls & $\mathrm{kg}$ & 4 & $4-5$ & Milling house and small scale food processer in DK & Peri urban \& urban fatteners \\
\hline 6 & Oat hulls & $\mathrm{kg}$ & 5 & $4-5$ & Milling house and small scale food processer in DK & Urban fatteners \\
\hline 7 & Grass pea hulls & $\mathrm{kg}$ & 5 & $4.5-5$ & Milling house and small scale food processer in DK & Peri urban \& urban fatteners \\
\hline 8 & Corn head \& cob & - & - & - & Farmers farm in DK & Peri urban fatteners only \\
\hline 9 & Pea hulls & $\mathrm{kg}$ & 5 & $4.5-5$ & Milling house and small scale food processer in DK & Urban fatteners \\
\hline 10 & Bean hull & $\mathrm{kg}$ & 5 & $4-5$ & Milling house and small scale food processer in DK & Urban fatteners \\
\hline 11 & Barley straw & Donkey capacity & 160 & $140-200$ & Farmers farm in DK & $\begin{array}{l}\text { Peri urban fatteners } \\
\text { dominantly }\end{array}$ \\
\hline 12 & Sorghum Stover & Per 'Kemer' & 250 & $250-450$ & Farmers farm + market around Kemisse & Peri urban \& urban fatteners \\
\hline 13 & Lentil hulls & $\mathrm{kg}$ & 5 & $5-5.50$ & Milling house and small scale food processer in DK & Urban fatteners \\
\hline
\end{tabular}


Table 7: Identified concentrate feed resources used for cattle fattening practices, price, sources and users in Dessie and Kombolcha towns

\begin{tabular}{|c|c|c|c|c|c|c|}
\hline & $\begin{array}{l}\text { dentified concentrate feed } \\
\text { resources }\end{array}$ & Unit & $\begin{array}{c}\text { Current } \\
\text { price (birr) }\end{array}$ & $\begin{array}{c}\text { Price range } \\
\text { (Birr) }\end{array}$ & Sources & Users \\
\hline 1 & Grass pea leftover ${ }^{\wedge}$ & $\mathrm{kg}$ & 6.50 & $6-8$ & Milling house and small scale food processer DK & $\begin{array}{l}\text { Peri urban \& urban } \\
\text { fatteners }\end{array}$ \\
\hline \multirow[t]{2}{*}{2} & $\begin{array}{l}\text { - Un processed corn grain } \\
\text { - } \quad \text { Processed corn grain }\end{array}$ & $\mathrm{kg}$ & 5.30 & $4-5.40$ & From farm and Market DK & $\begin{array}{l}\text { Peri urban \& urban } \\
\text { fatteners }\end{array}$ \\
\hline & & $\mathrm{kg}$ & 6.00 & $5.20-6.10$ & Feed market in DK & Urban fatteners \\
\hline \multirow[t]{2}{*}{3} & Dried brewers grain & per car & 2700 & 2700 & Kombolcha Beer factory (BGI ETHIOPIA) & Urban fatteners \\
\hline & & per sack & 125 & $120-130$ & Market in DK & Urban fatteners \\
\hline 4 & Wheat bran & $\mathrm{kg}$ & 4.70 & $3.80-5.20$ & Flour factory in DK & $\begin{array}{l}\text { Peri urban \& urban } \\
\text { fatteners }\end{array}$ \\
\hline 5 & Wet brewers grain & Per car & 2000 & 2000 & Kombolcha Beer factory (BGI ETHIOPIA) & Urban fatteners \\
\hline 6 & Oat grain & $\mathrm{kg}$ & 32 & $26-34$ & Feed market in DK & Urban fatteners \\
\hline 7 & Wheat middling & $\mathrm{kg}$ & 5.50 & $5-5.50$ & Flour factory in DK & $\begin{array}{l}\text { Peri urban \& urban } \\
\text { fatteners }\end{array}$ \\
\hline 8 & Molasses & & & & Tendaho sugar processing factory & Urban fatteners \\
\hline 9 & Wheat leftover^ & $\mathrm{kg}$ & 7 & $6.50-7.50$ & Milling house and small scale food processers in DK & Urban fatteners \\
\hline 10 & Flour factory leftover & $\mathrm{kg}$ & 5 & $2-5$ & Flour factory in DK & Urban fatteners \\
\hline 13 & Linseed cake & $\mathrm{kg}$ & 8 & $6-8$ & $\begin{array}{l}\text { Small scale oil seed processer in Dessie town and from } \\
\text { other towns dominantly }\end{array}$ & Urban fatteners \\
\hline 11 & Different feed left over & $\mathrm{kg}$ & 5 & $4-5$ & Flour factory in DK & $\begin{array}{l}\text { Peri urban \& urban } \\
\text { fatteners }\end{array}$ \\
\hline 12 & Noug seed cake & $\mathrm{kg}$ & 8 & $6-8$ & $\begin{array}{l}\text { Small scale oil seed processers in Dessie town and from } \\
\text { other towns dominantly }\end{array}$ & Urban fatteners \\
\hline 14 & Milling leftover & $\mathrm{kg}$ & 5 & $3-5$ & Milling house in DK & Urban fatteners \\
\hline 15 & Barley leftover^ & $\mathrm{kg}$ & 5 & $5-6$ & Milling house and small scale food processer in DK & $\begin{array}{l}\text { Peri urban \& urban } \\
\text { fatteners }\end{array}$ \\
\hline 16 & Bean leftover^^ & $\mathrm{kg}$ & 7 & $6.5-7$ & Milling house and small scale food processer in DK & Urban fatteners \\
\hline 17 & Lentils leftover ${ }^{\wedge}$ & $\mathrm{kg}$ & 7 & $6.50-7$ & Milling house and small scale food processer in DK & Urban fatteners \\
\hline 18 & Formulated ration & $\mathrm{kg}$ & 5.40 & $5-8$ & Government aid & $\begin{array}{l}\text { Peri urban and urban } \\
\text { fatteners }\end{array}$ \\
\hline 19 & Pea leftover^ & $\mathrm{kg}$ & 7 & $6.50-7$ & Milling house and small scale food processer in DK & Urban fatteners \\
\hline
\end{tabular}




\section{Seasonal Availability and Months of Distribution of the Identified Feed Resources:}

The seasonal availability and months of distribution of the overall identified feed resources is summarized in Table 8 and 9.

Table 8: Identified roughage and non -conventional feed resources used for cattle fattening, month of availability and distribution in Kombolcha and Dessie towns

\begin{tabular}{|c|c|c|c|c|c|c|c|c|c|c|c|c|c|c|c|}
\hline \multirow{2}{*}{\multicolumn{2}{|c|}{ Identified feed resources }} & \multirow[t]{2}{*}{ Local name (Amharic) } & \multirow{2}{*}{$\underset{8}{3}$} & \multicolumn{12}{|c|}{ Month of availability and distribution } \\
\hline & & & & Sep. & Oct. & Nov. & Dec. & Jan. & Feb. & Ma. & April & May & June & July & Aug. \\
\hline \multicolumn{16}{|c|}{ Green roughage } \\
\hline 1 & Green grass & Yewober sar & $\mathrm{C}$ & $* * *$ & $* * *$ & $* *$ & & & & & & & & & $* *$ \\
\hline 2 & Green maize Stover & Ertebi yebekolo ageda & $\mathrm{C}$ & $* *$ & $* * *$ & $*$ & & & & & & & & & $*$ \\
\hline 3 & Sugar can tops & Shenkora ageda chaf & $\mathrm{C}$ & & & & & $*$ & $*$ & $* *$ & $* *$ & $* *$ & $*$ & & \\
\hline 4 & Different weeds & Wokiya & $\mathrm{C}$ & $* *$ & & & & & & & & & & $*$ & $* *$ \\
\hline \multicolumn{16}{|c|}{ Dry roughage } \\
\hline 2 & Maize Stover & Yebekolo ageda & $\mathrm{C}$ & $*$ & $* * *$ & $* * *$ & $*$ & & & & & & & & $*$ \\
\hline 3 & Teff straw & chid & $\mathrm{C}$ & & $*$ & $*$ & $* *$ & $* *$ & $*$ & & & & & & \\
\hline 4 & Wheat straw & Yesende chid (geleba) & $\mathrm{C}$ & & & $* *$ & $* *$ & $* *$ & $* *$ & $*$ & & & & & \\
\hline 5 & Barley hulls & Yegebise geleba & $\mathrm{C}$ & & & & $* *$ & $* *$ & $* *$ & $*$ & $*$ & & & & \\
\hline 6 & Oat hulls & Yaja geleba & $\mathrm{C}$ & & & & $*$ & $*$ & $*$ & $*$ & $*$ & & & & \\
\hline 7 & Grass pea hulls & Yeguwaya geleba & $\mathrm{C}$ & $*$ & $* *$ & $* *$ & $* *$ & $* *$ & $*$ & $*$ & $*$ & $*$ & $*$ & & \\
\hline 8 & Corn head \& cob & Enbut bekolo & $\mathrm{C}$ & $*$ & $*$ & & & & & & & & & & $*$ \\
\hline 9 & Pea hulls & Yater geleba & $\mathrm{C}$ & & & $*$ & $*$ & $*$ & $*$ & $*$ & $*$ & $*$ & & & \\
\hline 11 & Barley straw & Yegebise chid (geleba) & $\mathrm{C}$ & & & & $* *$ & $* *$ & $* *$ & $*$ & $*$ & & & & \\
\hline 12 & Sorghum Stover & Yezengada ageda & $\mathrm{C}$ & & $*$ & $* *$ & $* *$ & $*$ & & & & & & & \\
\hline 13 & Lentil hulls & Yemeser geleba & $\mathrm{C}$ & & & & $*$ & $*$ & $*$ & & & & & & \\
\hline \multicolumn{16}{|c|}{ Non-conventional feed } \\
\hline 1 & Poultry litter (day old ) & Yedoro kus (yechachut) & $\mathrm{NC}$ & $* *$ & $* *$ & $* *$ & $* *$ & $* *$ & $* *$ & $* *$ & $* *$ & $* *$ & $* *$ & $* *$ & $* *$ \\
\hline 2 & $\begin{array}{l}\text { Poultry litter (layer \& } \\
\text { pullet) }\end{array}$ & Yedoro kus (enkulal tayi) & $\mathrm{NC}$ & $* *$ & $* *$ & $* *$ & $* *$ & $* *$ & $* *$ & $* *$ & $* *$ & $* *$ & ** & $* *$ & $* *$ \\
\hline 3 & Poultry litter (broiler) & Yedoro kus (ye sega) & $\mathrm{NC}$ & $* *$ & $* *$ & $* *$ & $* *$ & $* *$ & $* *$ & $* *$ & $* *$ & $* *$ & ** & $* *$ & $* *$ \\
\hline 4 & 'Atela' left over & Yetela atela & $\mathrm{NC}$ & $*$ & $*$ & $*$ & $*$ & $*$ & $*$ & $*$ & $*$ & $*$ & $*$ & & \\
\hline 5 & 'Korefe' leftover & Yekeorefe atela & $\mathrm{NC}$ & $*$ & $*$ & $*$ & $*$ & $*$ & $*$ & $*$ & $*$ & $*$ & $*$ & & \\
\hline
\end{tabular}


University leftover food

Yemegeb treferafi

$\mathrm{NC} \quad * \quad * \quad * \quad * \quad * \quad *$

$* * *=$ Abundantly available $* *=$ Moderately available $*=$ Less available $\mathrm{C}=$ Conventional $\mathrm{NC}=$ Non-conventional

Table 9: Identified concentrates feed resources used for cattle fattening, month of availability and distribution in Kombolcha and Dessie towns

\begin{tabular}{|c|c|c|c|c|c|c|c|c|c|c|c|c|c|c|c|}
\hline \multirow{2}{*}{\multicolumn{2}{|c|}{$\begin{array}{l}\text { Identified feed resources } \\
\text { Concentrates }\end{array}$}} & \multirow{2}{*}{\multicolumn{2}{|c|}{ 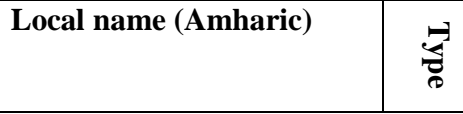 }} & \multicolumn{12}{|c|}{ Month of availability and distribution } \\
\hline & & & & Sep & Oc & No & De & Jan & Feb. & Ma. & Apr & May & Jun & July & Aug. \\
\hline 1 & Grass pea leftover $^{\wedge}$ & Yeguwaya duka ${ }^{\wedge}$ & $\mathrm{C}$ & $*$ & $* *$ & $* *$ & $* *$ & $* *$ & $*$ & $*$ & $*$ & $*$ & $*$ & & \\
\hline 2 & Corn grain & Bekole & $\mathrm{C}$ & $* *$ & $* *$ & $* * *$ & $* * *$ & $* * *$ & $* * *$ & $* *$ & $* *$ & $* *$ & $* *$ & $* *$ & $* *$ \\
\hline 3 & Dried brewers grain & Derek yebira chimaki & $\mathrm{NC}$ & $* *$ & $* *$ & $* *$ & $* *$ & $* *$ & $* *$ & $* *$ & $* *$ & $* *$ & $* *$ & $* *$ & $* *$ \\
\hline 4 & Wheat bran & Yeferes furishika & $\mathrm{C}$ & $*$ & $* * *$ & $* * *$ & $* * *$ & $* *$ & $* *$ & $*$ & $*$ & $*$ & $*$ & $*$ & $*$ \\
\hline 5 & Wet brewers grain & Ertib yebira chimaki & $\mathrm{NC}$ & $* *$ & $* *$ & $* *$ & $* *$ & $* * *$ & $* *$ & $* * *$ & $* *$ & $* *$ & $* *$ & $* *$ & $* *$ \\
\hline 6 & Oat grain & Aja & $\mathrm{C}$ & & & & $*$ & $*$ & $*$ & $*$ & $*$ & & & & \\
\hline 7 & Wheat middling & furshikelo & $\mathrm{C}$ & $*$ & $* * *$ & $* * *$ & $* * *$ & $* *$ & $* *$ & $*$ & $*$ & $*$ & $*$ & $*$ & $*$ \\
\hline 8 & Molasses & - & $\mathrm{C}$ & - & - & - & - & - & - & - & - & - & - & - & - \\
\hline 9 & Wheat leftover ${ }^{\wedge}$ & Yesende duka^ & $\mathrm{C}$ & $*$ & $*$ & $*$ & $*$ & $*$ & $*$ & $*$ & $*$ & $*$ & $*$ & $*$ & $*$ \\
\hline 10 & Flour factory leftover & Yeduket fabrica bitary & $\mathrm{C}$ & $*$ & $*$ & $*$ & $*$ & $*$ & $*$ & $*$ & $*$ & $*$ & $*$ & $*$ & $*$ \\
\hline 13 & Linseed cake & fagulo ye telba & $\mathrm{C}$ & & $*$ & $*$ & $*$ & & & & & & & & \\
\hline 11 & Different feed left over & Entiritaryi & $\mathrm{C}$ & $*$ & $*$ & * & $*$ & * & * & * & $*$ & * & $*$ & * & $*$ \\
\hline 12 & Noug seed cake & fagulo ye nuge & $\mathrm{C}$ & & $*$ & $*$ & $*$ & & & & & & & & \\
\hline 14 & Milling leftover & Yewoficho bet tiragi & $\mathrm{C}$ & $*$ & $*$ & $*$ & $*$ & $*$ & $*$ & $*$ & $*$ & $*$ & $*$ & $*$ & $*$ \\
\hline 15 & Barley leftover ${ }^{\wedge}$ & Yegebise duka & $\mathrm{C}$ & & & & $* *$ & $* *$ & $* *$ & * & $*$ & & & & \\
\hline 16 & Bean leftover ${ }^{\wedge}$ & Yebakela duka^ & $\mathrm{C}$ & & & $*$ & $*$ & $*$ & $*$ & $*$ & $*$ & $*$ & & & \\
\hline 17 & Lentils leftover ${ }^{\wedge}$ & Yemeser duka^ & $\mathrm{C}$ & & & & $*$ & $*$ & $*$ & & & & & & \\
\hline 18 & Formulated ration & Yetemetatene meno & $\mathrm{C}$ & & & & & & & $* *$ & $* *$ & $* *$ & $* *$ & $*$ & \\
\hline 19 & Pea leftover^^${ }^{\wedge}$ & Yater duka^ & $\mathrm{C}$ & & & $*$ & $*$ & $*$ & $*$ & $*$ & $*$ & $*$ & & & \\
\hline
\end{tabular}

$* * *=$ Abundantly available $* *=$ Moderately available $*=$ Less available ${ }^{\wedge}$ leftover/ duka $^{\wedge}=$ Occasional surplus or damaged grain during processing

$\mathrm{C}=$ Conventional $\mathrm{NC}=$ Non-conventional 


\section{General Limitations and Challenges Related to Feed Resources Used for Cattle Fattening:}

Limited access of feed resources, difficulties related to transportation and cost to collect different feeds from different feed suppliers, recurrent drought, feed shortage, price increment and unpredictable feed cost variation, lack of governmental feed processing factories and suppliers, limited brewery grain access, poor distribution of the available feed resources, illegal cattle feed traders and difficulty to access molasses were pronounced challenges and limitations reported by cattle fatteners in Dessie and Kombolcha towns.

\section{Chemical composition of selected feed ingredients}

Chemical composition of selected feed ingredients used for cattle fattening in Dessie and Kombolcha towns is explained in Table 10.

Table 10: Chemical composition of selected feed ingredients used for cattle fattening in Dessie and Kombolcha towns

\begin{tabular}{|c|c|c|c|c|c|c|c|c|c|}
\hline \multirow[t]{3}{*}{ Feed ingredients } & \multicolumn{7}{|c|}{ Chemical composition } & \multirow[b]{2}{*}{$\mathrm{HC}$} & \multirow[b]{2}{*}{$\bar{C}$} \\
\hline & DM & Ash & $\mathbf{O M}$ & $\mathbf{C P}$ & NDF & ADF & $\overline{\text { ADL }}$ & & \\
\hline & \multicolumn{7}{|c|}{$\% \mathrm{DM}$} & & \\
\hline $\begin{array}{l}\text { Natural grass hay } \\
(\mathrm{NGH})\end{array}$ & 95.29 & 8.59 & 86.7 & 4.79 & 58.29 & 42.41 & 6.23 & 15.88 & 36.18 \\
\hline Dried breweries grain & & & & & & & & 32.42 & 15.62 \\
\hline (DBG) & 89.69 & 4.45 & 85.24 & 31.39 & 56.21 & 23.79 & 8.17 & & \\
\hline Poultry litter (layer) & 95.86 & 12.56 & 83.3 & 23.33 & 27.19 & 22.7 & 7.65 & 4.49 & 15.05 \\
\hline Poultry litter (pullet) & 95.77 & 10.87 & 84.9 & 22.33 & 26.4 & 21.31 & 7.26 & 5.09 & 14.05 \\
\hline Poultry litter (day old) & 95.83 & 9.75 & 86.08 & 23.36 & 26.16 & 22.02 & 8.33 & 4.14 & 4.19 \\
\hline $\begin{array}{l}\text { Grass pea leftover } \\
\text { (GPLO) }\end{array}$ & 92.00 & 9.35 & 82.65 & 7.64 & 62.5 & 45.47 & 8.51 & 17.03 & 36.96 \\
\hline Processed corn grain & & & & & & & & 11.39 & 1.36 \\
\hline (PCR) & 93.70 & 1.61 & 92.09 & 7.59 & 16.16 & 4.77 & 3.41 & & \\
\hline Wheat middling (WM) & 92.34 & 4.17 & 88.17 & 15.37 & 39.07 & 15.26 & 5.23 & 23.81 & 10.03 \\
\hline Wheat bran (WB) & 90.43 & 5.7 & 84.73 & 16.73 & 43.12 & 14.81 & 3.82 & 28.31 & 10.99 \\
\hline
\end{tabular}

$\mathrm{ADF}=$ Acid detergent fiber; $\mathrm{ADL}=$ Acid detergent lignin; $\mathrm{CP}=$ Crude protein; $\mathrm{DM}=$ Dry matter; $\mathrm{NDF}=\mathrm{Neutral}$ detergent fiber, $\mathrm{HC}=$ Hemicellulose, $\mathrm{C}=$ Cellulose

\section{Discussions:}

\section{Identified Feed Resources used for Fattening Cattle in Dessie and} Kombolcha Towns:

Those identified feed resources were used as major and supplementary feeds by the interviewed cattle fatteners which grouped further into three categories: viz; (i) green roughages, (ii) dry roughages and (iii) concentrates. The green roughage category, which included green grass which is native wet grass comes from the adjacent farm land, locally called 'wober' or pasture land, green maize stover, sugar can tops and different weeds were ranked $1^{\text {st }}, 2^{\text {nd }}, 3^{\text {rd }}$ and $4^{\text {th }}$, respectively, based on availability and abundance in both study towns. Particularly, green grasses, different weeds, 
green maize stover were predominantly available for peri urban cattle fatteners and farmers who have pasture land. The indicated feeds were difficult to acquire at market level unless otherwise purchased from farmers farm and pasture lands in green form (Table 1).

In the category of dry roughage, hay, maize stover, teff straw, wheat straw, barley hulls, oat hulls, grass pea hulls, corn head and cob, pea hulls, bean hulls, barley straw, sorghum stover and lentil hulls, were ranked $1^{\text {st }}$ to $13^{\text {th }}$ based on availability and abundance in Dessie town, respectively, whereas, hay, maize stover, sorghum stover, teff straw, grass pea hulls, pea hulls, wheat straw, barley hulls, oat hulls, bean hulls, barley straw, lentil hulls, and corn head \& cob were ranked $1^{\text {st }}$ to $13^{\text {th }}$ based on availability and abundance in Kombolcha town, respectively (Table 1). The current results agreed with Zewdie (2010) who reported that teff and wheat straws are important sources of livestock feed in the highland. Barley and oat straws are also important and accessible in the area. Straw supplementation is commonly restricted to work-oxen and lactating cows.

Underneath non-conventional feed resources category, poultry litter from layer house, poultry litter from day old chicken house, (Figure 9) poultry litter from broiler house, 'Atela' left over, 'Korefe' leftover and university leftover food were ranked $1^{\text {st }}$ to $6^{\text {th }}$, respectively, based on availability and abundance in Dessie and Kombolcha towns (Table 1). From concentrate feed category, wheat bran, corn grain, dried brewers grain, wet brewers grain, wheat middling, grass pea leftover, (Figure 9) oat grain, pea leftover, wheat leftover, flour factory leftover, linseed cake, different feed left over, noug seed cake, milling leftover, barley leftover, bean leftover, lentils leftover, formulated ration and molasses were ranked $1^{\text {st }}$ to $19^{\text {th }}$ based on availability and abundance in their logical order in Dessie town. Although, in the case of Kombolcha town, from concentrate category, corn grain, wet brewers grain, dried brewers grain, wheat bran, grass pea leftover, wheat middling, oat grain, pea leftover, barley leftover, wheat leftover, flour factory leftover, different feed left over, formulated ration, molasses, milling leftover, lentil leftover, bean leftover, linseed cake and noug seed cake were ranked $1^{\text {st }}$ to $19^{\text {th }}$ in their logical order based on availability and abundance (Table 2). 'Duka' is leftover and occasional surplus or damaged grain during processing. The current findings and feed resources classification agree with Younas and Yaqoob, (2015) who indicated that green roughages included fodder crops, grasses including shrubs and forbs, sugar beet tops, sugarcane tops, silages and tree leaves. Dry roughages included hay, straws, stover's and hulls. Concentrates included cereal grains, oilseed cakes and meals, cereal brans and polishing's, molasses and sugar beet pulp. Nonconventional feed resources have the potential to play a major role in meeting the feed shortage in the years to come. 


\section{Classifications of the Identified Feed Resources Based on Significance for Cattle Fattening in Dessie and Kombolcha Towns}

Thus, feed resources categorized under $1^{\text {st }}$ choice were hay, poultry litter comes for day old chicken house, layer house, pullet house and broiler house, grass pea leftover, corn grain, dried brewers grain, wet brewers grain, wheat bran, bean leftover, formulated ration, wheat leftover, and pea leftover in both study towns. As a $2^{\text {nd }}$ choice cattle fattener were picks green grass, green maize stover, sugar can tops, maize stover, teff straw, barley hulls, oat hulls, grass pea hulls, oat grain, wheat middling, molasses, different feed leftover, barley leftover and linseed cake in both study towns. In the $3^{\text {rd }}$ position, different weeds comes from farm, wheat straw, corn head \& cob, pea hulls, bean hulls, barley straw, sorghum stover, lentil hulls, 'Atela' left over, 'Korefe' leftover, university leftover food, flour factory leftover, noug seed cake, milling leftover and lentils leftover in both study towns. The identified feed resources classification were totally similar in both towns, this is due to cattle fatteners in both towns use similar cattle market, and such condition creates media to sharing experience one from the other.

Based on the report of cattle fatteners and their practical experiences, those feed resources listed under first choice were mandatory and must be incorporated in the cattle fattening ration for their significance and importance in nutritional values. They explained that feed resources ranked in the $1^{\text {st }}$ order have potential to fatten cattle within short period of time. When the condition was not suitable to full fill or incorporate the first choice feed resources in the ration or if they missed some of the first choice feed ingredients, they were used feed resources ranked in the second order via considering the cost and availability of the feeds. Particularly, cattle fatteners were incorporate those feed resources categorized under $3^{\text {rd }}$ choice to increase the bulkiness or quantity of the ration planned to give for a given number of cattle. Generally, in both study towns cattle fatteners reported that feed resources selection was highly dependable with the availability and price of each ingredient. At a time of no alternatives, particularly urban cattle fatteners were forced to purchase and use any of the feed categories accessible in the market.

\section{Potential Feed Suppliers:}

Poultry farms, flour factors, milling houses, micro and small food processors, local brewery houses, farmers, brewery factory, oil seed processers, pasture lands, traders, sugar processing factory, university and government aid were the identified cattle feed sources in Dessie and Kombolcha towns. 
Poultry farms: Two poultry farms used as poultry litter sources were identified in Dessie and Kombolcha towns, namely, Kombolcha poultry farm which is governmental enterprise is found in Kombolcha town and Dessie Gredado private poultry farm found in Dessie town. In addition, in both study towns there were private small scale organized poultry farms used as supplementary poultry litter sources for cattle fattening purpose which were structured by Micro and Small Enterprise Office. Furthermore, via feed trader's cattle fatteners were accessed poultry litter from Bishoftu town. Therefore, layer poultry litter, pullet poultry litter, day old poultry litter, and broiler poultry litter were the identified non-conventional feed resources used by cattle fatteners in both study towns (Figure 1). The current finding similar with Asrat et al., (2008) which reported that poultry litter has been identified as one of the non-conventional feeds for ruminant production.

Local brewery houses: Local brewery houses were one of the identified feed sources in both study towns. Under non-conventional feeds 'Atela' left over and 'Korefe' leftover were the two identified feed resources comes from local brewery houses in both study towns which is used for cattle fattening purpose. There are two types of 'Atela' left over namely, 'diffedef' the solid part which is extracted before the main product and 'kerery' the residue watery portion. Similarly, 'Korefe' leftover has two portions, viz., 'diffedef' the solid part which is extracted before the main product, and 'kereryi' residue of the watery portion (Figure 2). The current result agree with Zewdie (2010) reported that non-conventional feeds such as 'Atela' is not available at large and their contribution to livestock feed is small but used as a cooping strategy.

University output: University leftover food was another identified non-conventional feed resource in Dessie and Kombolcha towns. Such feed comes from Wollo University, especially those cattle fatteners and livestock holders near the university campus use such feed resources for fattening cattle and other livestock's. Due to limited availability few fatteners only get the services. The problem related to such feed resources reported by cattle fatteners were unless otherwise the feed resources dried properly it creates blotting on fattening cattle. 


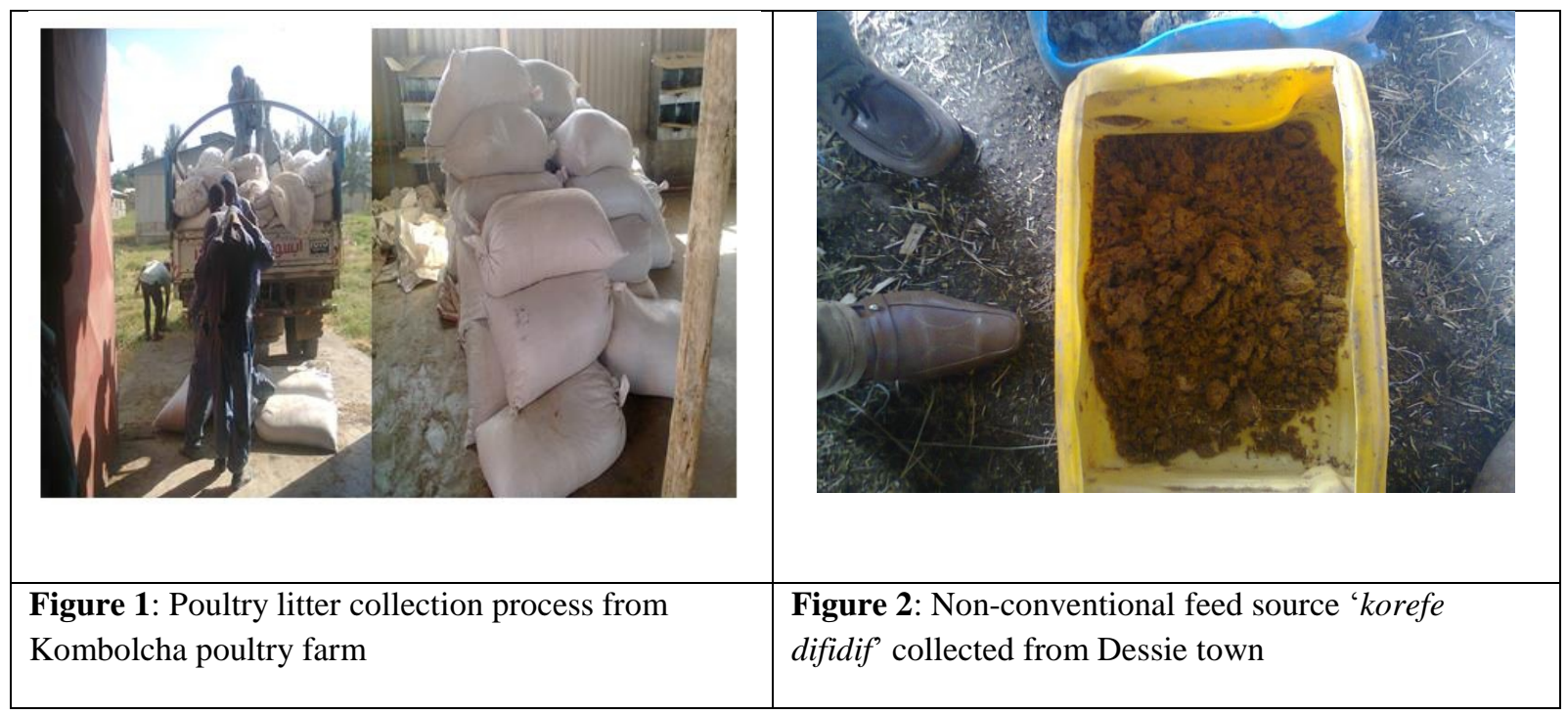

Flour factories: Flour factories were the major and dominate feed sources identified in both study towns for fattening cattle. There are 6 and 2 large flour factors in Dessie and Kombolcha towns, respectively (Table 3). Wheat middling, wheat bran, factory leftover were the output of the factories and dominate, common and major feed resources used for cattle fattening in both study towns (Figure 3). The current result disagrees with report of Firew and Getenet, (2010) which clarify that use of concentrate and agroindustrial by-products as livestock feeds especially for fattening and dairy is not common. Generally, in both study towns, individual and organized groups of urban and peri urban cattle fatteners were used the byproduct as major, common and dominate supplements for cattle fattening.

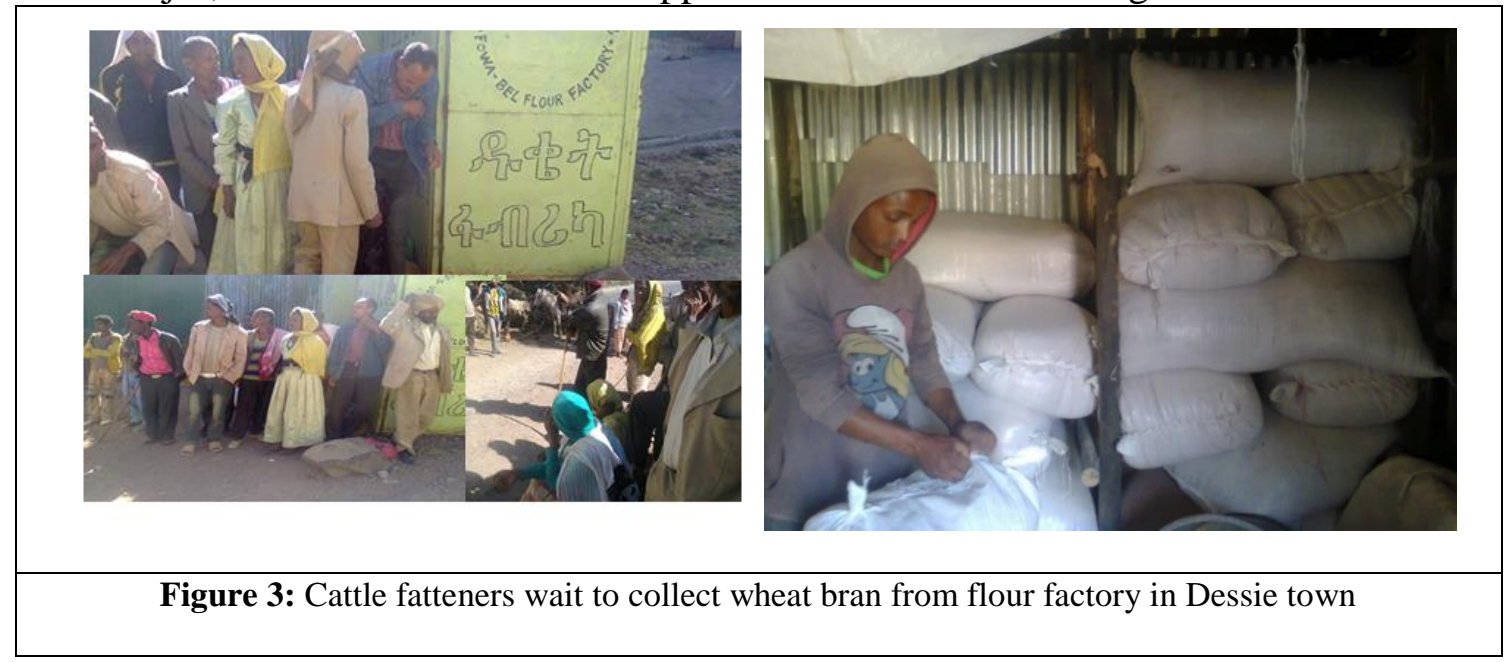


Micro and small food processors and milling houses: Private micro and small food processors and milling houses were other potential feed sources identified in both study towns. There were 18 and 36 micro and small private food processors, and 34 and 36 milling houses in Dessie and Kombolcha towns, respectively (Table 4). Particularly, barley hulls, oat hulls, grass pea hulls, pea hulls, bean hulls, lentil hulls, grass pea leftover, corn grain, wheat leftover, bean leftover, (Figure 9), different feed left over, milling leftover, barley leftover, lentils leftover, pea leftover were comes from micro and small food processors and milling houses. The limitations related to the indicated by products was transport challenge due to small but frequent supply of the feed resources. It needs to check the door of micro and small food processors and milling houses frequently to acquire the required amount. Due to the indicated reasons and difficulties the product was mostly accessible and used the product as major and supplements to urban cattle fatteners than peri urban cattle fatteners in both study towns. Cattle fatteners in both study areas were use ' $D u k a$ ' dominantly than hulls due to higher nutritive value. 'Duka' is leftover and occasional surplus or damaged grain during processing. Mainly cattle fatteners were used different feed resources hulls for the purpose to increase palatability and feed intake. For example, cattle fatteners were reported that oat hulls increase feed intake due to its taste nature. In addition, barely hull is used for the purpose of odor. Generally, processed feed resources has odor which leads cattle to take more. The current finding is agree with Firew and Getenet (2010) which reported that small-scale local mills in one way or another have contribution in supplying varieties of grain by-products. Pulse hulls are commonly used as feed for animals.

Governmental aid: Governmental aid was one feed sources for fattening cattle recognized during the study in both towns. As per the group discussion, when there was occurrences of sever feed shortage the government provide formulated ration for those drought affected groups. During such time the feed resources were available in the market. The limitation of such feed source was inadequate and irregular availability

Oilseed processer: Only one small scale oil seed processer which has a capacity to produce 120 quintal per year was identified in Dessie town (Table 4). Linseed cake, and nug seed cake were the output comes from the indicated oil seed processer. Generally, except the indicated sources there was no potential oil seed processor due to absence of oil seed production and agronomic practices in both study towns. The current result agrees with Firew and Getenet (2010) which reported that in Amhara region, there are many small-scale oil mills, which notably contribute to the oilseed cake 
supplies. Despite that, their contribution is declining from year to year due to increased price and decreased availability of oilseeds.

Feed traders: Feed traders were other main feed sources suppliers identified in both study towns. Oat grain, poultry litter and corn grain comes from feed traders. Particularly, in both study towns there was ample amount of corn grain with minimum price throughout the year and corn grain categorized as main crop and produced broadly in and around both the study areas. As well as through feed traders' abundant corn gains were available in trader houses in both study towns. Per quintal pure corn costs 400 - 540 birr (Table 7). Therefore, almost all of the cattle fatteners in both study areas use corn grain as a major and dominate feed supplement. On the other hand, oat grain is limited due to absence of strong agronomic practices in and around both study areas. In addition, even if the feed resources were preferred primary by cattle fatteners the price is very expensive when compare to other locally available feed resources (Table 7). Feed traders were used as a source for sugar can tops but the availability of the product was limited. Due to this reason less cattle fatteners use the product. At study town level relatively in Kombolcha town there was better accessibility of sugar can tops than Dessie town.

Sugar factory: Sugar factory was another identified feed source which provides molasses comes from Tendaho sugar factory. The limitation to use the indicated feed resources was difficulty of transport cost at individual cattle fattener level and lack of cooperatively with in cattle fatteners in both study towns.

Brewery factory: Kombolcha brewery factory was the only identified source for wet and dried brewery grains in and around the study towns which is established in Kombolcha town. Dried brewers grain, and wet brewers grain were the byproducts of the factory. Dessie and Kombolcha towns are also a beneficiary of the by-products of the brewery factory (Figure 4 and 5). The current result in line with (McDonald et al., 2002) who reported that, brewers dried grains is a good source of protein for fattening cattle in addition to their energy value. Because of its bulky, fibrous nature and low energy content, BDG (Dried Brewery Grains) is more suitable for ruminants than non-ruminants. It is not highly palatable, therefore; it should be mixed with feeds of better palatability. The supply of BSG (Brewery Spent Grains) could not satisfy the demands of the farmers found in Dessie and Kombolcha towns. This finding agrees with Firew and Getenet, 2010 who explained limited supply of the by product. The limitation of the product were limited access due to presence of high demand and particularly 
the wet brewery grain unless otherwise stored properly it creates bad odor with in the community.

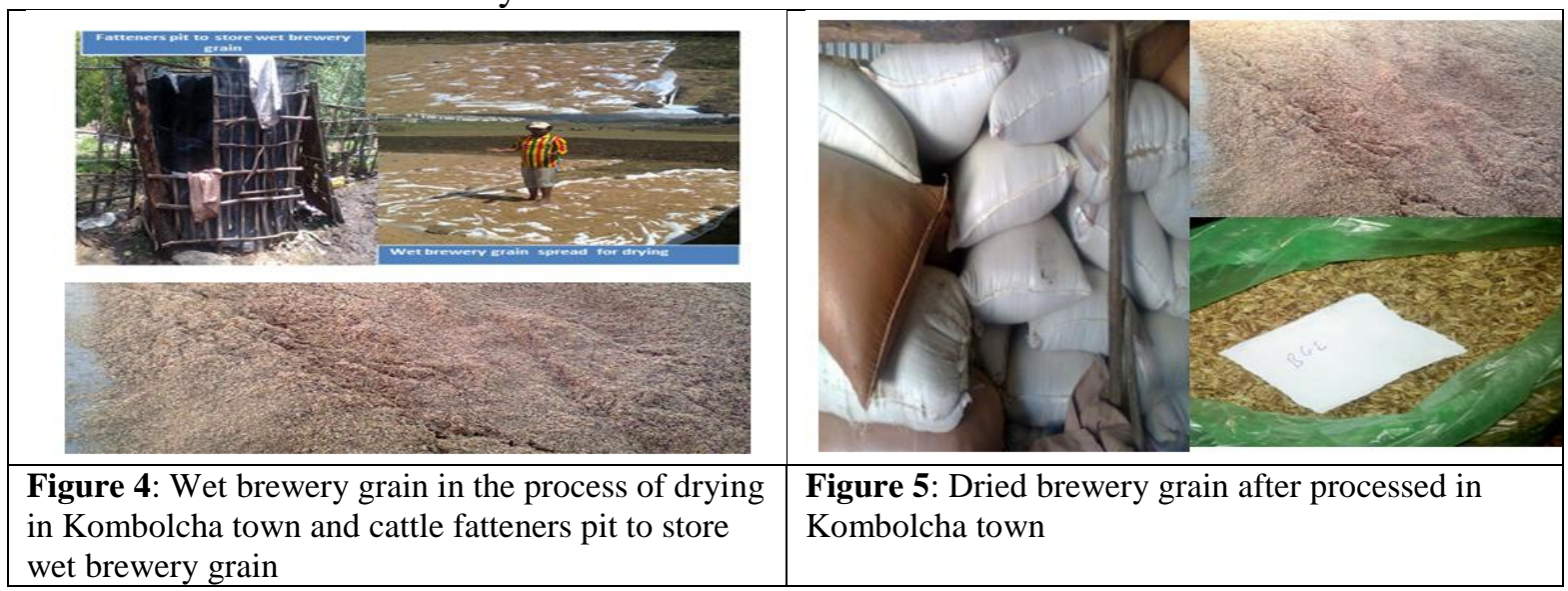

Small holder farmers and pasture lands: Small holder farmers and pasture lands were the major dry and green roughage feed sources identified in Dessie and Kombolcha towns. Green maize stover (Figure 6), different weeds comes from farmers farm, green grass is native wet grass comes from the adjacent farm land (Figure 7), hay, dried maize stover, teff straw, wheat straw, corn head \& cob, barley straw, sorghum stover were the identified feed resources comes from such feed source in both study towns. The limitation of such feed resources was except hay, majority of the feed resources mostly accessible for farmers and peri urban cattle fatteners who have pasture land. In addition, it is difficult to access at market level unless otherwise purchase the resources from the pasture land or farmers farm as its wet base. Due to limited availability of such feed resources cattle fatteners' particularly urban cattle fatteners was focused on hay as roughage feed sources.

\begin{tabular}{|l|l|l|}
\hline & \\
\hline $\begin{array}{l}\text { Figure 6: One of green roughage (green } \\
\text { corn) collected from Dessie town }\end{array}$ & $\begin{array}{l}\text { Figure 7: Native green grass in the process } \\
\text { of harvesting nearby Wollo University, } \\
\text { Dessie town }\end{array}$ & $\begin{array}{l}\text { Figure 8: Native grass harvested } \\
\text { and piled in the form of hay nearby } \\
\text { Wollo University, Dessie town }\end{array}$ \\
\hline
\end{tabular}


In general, hay is dominant dry roughage feed based on availability and abundance which is used for cattle fattening in Dessie and Kombolcha towns. Hay relatively available with minimum price September to December when compare to other months (Figure 8). The current finding in line with Zewdie (2010) which explain hay is commonly used way of feed preservation technique in Ethiopia which is expected to mitigate problems of livestock feeding during the dry period and therefore such experience is a good indicator that feeds are being efficiently utilized.

\section{Seasonal Availability and Months of Distribution of the Identified Feed Resources:}

As per group discussion like other areas in both study towns there was high seasonal variability of feed availability and distribution. This is due to seasonal agronomic practices, limited supply, and inbalancement of demand and supply and transport difficulties. As a whole, in the current both study towns specially September to December relatively there was abundant feed resources. On the other hand, the feed shortage and price increased severely towards January to June. During this period the scarcity and the feed price increased in alarming rate. This finding support the idea which shows alternating periods of surplus and deficit animal feed result in a very low level of production for the entire year (Aklilu 2004) and the current finding similar to Getachew (2002) reported that the quantity of feed is inadequate in the dry season for the existing livestock while there is surplus in the wet season. Similarly, agro industrial by products comes from flour factories such as wheat middling, wheat bran, and factory leftover were abundantly available throughout the year relative to other feed resources in both study towns. Particularly, November to December the feed resources were abundantly available with minimum price (Table 7 and 9). This is due to the indicated months were the major period to harvest different crops in and around both the study areas which will be inputs for factories. Consistently, in the peri urban kebeles farmers particularly, cattle fatteners were used their own feed resources come from the farm. According to Tessema et al., (2003), seasonal variations in feed quality and quantity are the main limitation to animal production and cause fluctuation in productivity throughout the year, particularly in the dry seasons during which feed is limited. In addition, feed resources comes from micro and small food processors and milling houses show great variability in accessibility, particularly grass pea leftover, relatively available. This is because food processors use mostly grass pea for the preparation of human food called 'shuro' due to its reasonable cost than other ingredients.

Therefore, grass pea leftover takes the dominant share and easily available as compere to other feed resources. Consistently, through 
considering the price, availability and degree of importance cattle fatteners were provided different grade for different feed resources comes from such micro and small food processors and milling houses sources. Hay is mostly available in abundance from September to November in both study towns when compare to other months. The indicated months particularly October to November was a major harvesting season. Commonly urban cattle fatteners were collect green grass via bid from different governmental and nongovernmental organization such as schools, hospitals, farmers and private owner which has pasture land. Generally, except dried brewery grain in the current both study towns' majority of the identified feed resources show great variation in availability and price due to limited supply and transportation problem. Particularly, oil seed cakes were greatly limited throughout the year with high cost.


Grass pea leftover



Wheat middling

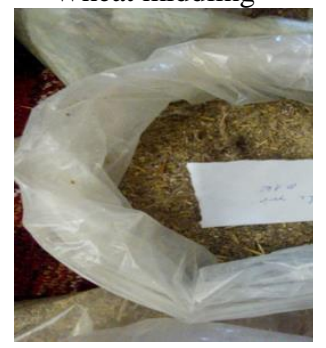

Poultry litter (layer)


Poultry litter (day old)

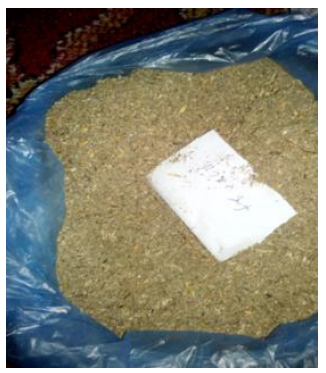

Barely left over

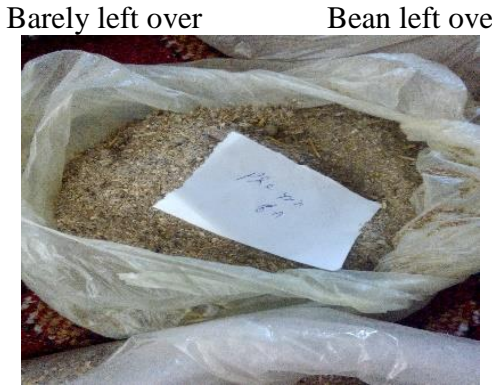

Poultry litter (pullet)

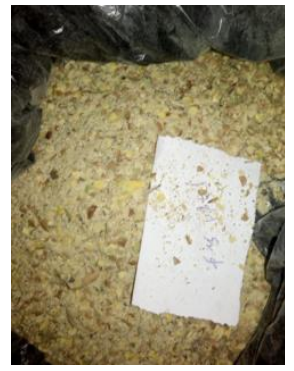

Bean left over

Figure 9: Some of the identified feed resources used for cattle fattening in Dessie and Kombolcha towns

\section{General Limitations and Challenges Related to Feed Resources}

Limited access of feed resources, difficulties related to transportation and cost to collect different feeds from different feed suppliers, recurrent drought, feed shortage, price increment and unpredictable feed cost variation, lack of governmental feed processing factories and suppliers, limited brewery grain access, poor distribution of the available feed resources, illegal cattle feed traders and difficulty to access molasses were pronounced challenges and limitations reported by cattle fatteners in Dessie and Kombolcha towns. The current result is in line with Firew (2010) which 
reported that inadequate feed supply is one of the major constraints hampering market-oriented livestock development in the Amhara National Regional State (ANRS) in particular and in Ethiopia in general. Similarly, Belete et al., (2010) explain the cattle fattening practice is constrained by high feed cost, poor quality and low availability of feed resources. Therefore, producing a high quantity and quality of feed for animals is a key factor in raising healthy and productive livestock sector (Bezahegn, 2014). Firew and Getnet (2010) reported that feed processing plants in Ethiopia are very few and still are not producing to their full capacity. Most of them are concentrated around the main highway between Addis Ababa and Adama.

\section{Conclusion}

To boost up the newly emerging urban as well as peri urban cattle fattening sector, feed shortage problem solving activities should get the first and foremost considerations. Therefore, to alleviate and minimize feed shortage, the effective approach is to focus on and due emphasis the use of locally available, identified potential feed resources in both towns. Predominantly, for effective and economical fattening program proper usage of the identified and potential feed resources should be the main thought. Accordingly from the identified 42 feed resources 9 feeds were highlighted with their chemical composition (first choices) based on availability, significance, current and future production potentials, accessibility and quantity to be released, supplier's actual potential to deliver, minimum and maximum price range and also based on cattle fatteners practical experience of both study towns. The proposed feed resources were corn grain, wheat middling, wheat bran, dried and wet brewers grain, hay, day old poultry litter, layer and pullet poultry litter, broiler poultry litter, and grass pea leftover. As a result, to run effective, profitable, economical cattle fattening program and to boost up the newly emerging urban as well peri urban cattle fattening practices in Dessie and Kombolcha towns, it is recommendable to focus on the proposed feed resources via considering their nutritive values.

\section{Recommendations:}

To minimize feed shortage difficulty in both study towns the following proposed solutions were forwarded:

- It is valuable to advice cattle fatteners to collect available feed resources and enriched their feed bunk before the feed scarcity and price increment observed with strong extension service.

- As immediate remedy, Agriculture Offices in both study towns should take action to balance feed demand and supply via analysis and coordination of feed suppliers with cattle fatteners. Additionally, for 
proper distribution and usage of poultry litter and brewery grain peri urban and urban cattle fatteners should be organized and scheduled according to their feed demand and supply.

- The government should initiate private micro and small feed processing enterprises to be part of the sector. In addition, governmental cattle feed processing plant should be established as a long term remedy.

- Hay preparation as well as use of poultry litter experience of urban and peri urban cattle fatteners should be supported and strengthened with strong extension service.

- Broadly, there should be functional linkage between stakeholders such as different governmental offices (particularly Agriculture and Micro and Small Enterprise Offices), urban and peri urban cattle fatteners and feed suppliers in both study towns.

\section{Acknowledgements:}

The authors would like to acknowledge College of Veterinary Medicine and Agriculture of Addis Ababa University and Arba Minch University, Ethiopia for funding the study. We also acknowledge the urban and peri urban cattle fatteners, Agricultural Experts, Development Agents in Dessie and Kombolcha towns, Micro and Small food processors, Oil seed processers, Local brewery houses, Kombolcha BGI Ethiopia, Poultry farms, Milling houses, Traders, Flour factories, Micro and Small Enterprise Office, Agriculture office and Trade and Industry Coordination Office of Dessie and Kombolcha towns for their willingness to provide the necessary information.

\section{References:}

1. Adugna T. (2007). Feed resources for producing export quality meat and livestock in Ethiopia, examples from selected Woredas in Oromia and SNNP regional states. Ethiopia Sanitary and Phytosanitary Standards and Livestock and meat Marketing Program (SPS-LMM). USAID, Ethiopia.

2. Aklilu W. (2004). Fattened Animal Marketing System Study. Agricultural Commodity Marketing System Study Project, Amhara National Regional State Head of Government Office, Final Report, Annex 13.

3. Tsadik A, Tamir B, and Melaku S. (2008). Inclusion of different proportions of poultry litter in the rations of yearling Hararghe Highland goats. Livestock Research for Rural Development. Volume 20, Article no 48. Retrieved August 24, 2016,from http://www.lrrd.org/lrrd20/3/tsad20048.htm.

4. Belete A, Azage T, Fekadu B, and Berhanu G. (2010). Cattle milk and meat production and marketing systems and opportunities for 
market-orientation in Fogera woreda, Amhara region, Ethiopia. IPMS (Improving Productivity and Market Success) of Ethiopian Farmers Project Working Paper 19. ILRI (International Livestock Research Institute), Nairobi, Kenya. 65 pp.

5. Bezahegn A. (2014). Small Scale Beef Cattle Fattening Practices, Onfarm Performance Evaluation And Opportunities For Market Orientation In Western Hararghe Zone, Chiro District, M.Sc. Thesis.

6. Dawit B. (2013). Economic and Social Vulnerability Of Rural Urban Migrant Women in Dessie Town, South Wollo Zone, Amhara Regional State, M.A Thesis.

7. Eskinder Z, Eyassu Y, and Mitiku H. (2010) Assessment of the impact of industrial effluents on the quality of irrigation water and changes on soil characteristics (a case of Kombolcha town), fourteenth international water technology conference, iwtc 14, 2010, Cairo, Egypt.

8. Firew T, and Getnet A (2010). Feed resource assessment in Amhara National Regional State. September, 2010 Addis Ababa.

9. Getachew E. (2002). An Assessment of Feed Resources, their Management and Impact on Livestock Productivity in Ginchi Watershed Area. M. Sc. Thesis. Alamaya University of Agriculture, Alemaya. $172 \mathrm{pp}$.

10. Habib G and Siddiqui M (1994). Feeds and Feeding in Animal Husbandry. S I Shah (Editor), TIPAN Project of NWFP Agricultural University, Peshawar published by NBF, Islamabad.

11. http://en.wikipedia.org/wiki/Dessie, retrieved in December 2014

12. McDonald P, Edwards R, Greenhalgh J \& Morgan, C. (2002). Animal nutrition, 6th Edition, Prentice Hall, London, pp.471-581.

13. Muluwork Z. (2014). An assessment of livelihood and food security of farmers displaced due to urban expansion, the case of Kombolcha town in Amhara national regional state, Ethiopia, a thesis report.

14. Samuel T. (2012). Effects of supplementing cassava leaf meal, brewers' dried grain and their mixture on growth and carcass traits of local goats fed urea treated tef straw, Haramaya University M.Sc. thesis.

15. Seyoum B, Getnet A, Abate T. (2001). Present status and future direction in feed resources and nutrition research targeted for wheat based crop-livestock production system in Ethiopia. Wheat and weeds: food and feed. Proceedings of the two stake holder workshops, santa cruz, bolivia.

16. Tessema Z, Aklilu A and Ameha S. (2003). Assessment of the Livestock Production System, Available Feed Resources and 
marketing situation in Belesa Woreda: A case study in drought prone areas of Amhara Region.

17. Tilahun A, Solomon M, and Roothaert R. (2005). Intensification of Livestock Feed Production in Ethiopian Highlands: Potential and Experiences of the African Highlands Initiative. Presented at the 19th EVA Annual conference, 8 June 2005, Addis Ababa, Ethiopia.

18. Younas M and Yaqoob M. (2005) Feed resources of livestock in the Punjab, Pakistan. Livestock Research for Rural Development. Vol. 17, Art. \#18. Retrieved August 24,2016,fromhttp://www.lrrd.org/lrrd17/2/youn17018.htm

19. Zewdie W. (2010). Livestock production systems in relation with feed availability in the highlands and central rift valley of Ethiopia Haramaya University MSc.Thesis. 